CARNETS DE Carnets de géographes

GÉOGRAPHES.

$7 \mid 2014$

Les espaces de l'entre-deux

\title{
Explore Everything : Place-Hacking The City
}

\section{Louise Dorignon}

\section{(2) OpenEdition}

Journals

Édition électronique

URL : http://journals.openedition.org/cdg/507

DOI : $10.4000 /$ cdg. 507

ISSN : 2107-7266

Éditeur

UMR 245 - CESSMA

Référence électronique

Louise Dorignon, «Explore Everything : Place-Hacking The City », Carnets de géographes [En ligne], 7| 2014, mis en ligne le 01 décembre 2014, consulté le 24 septembre 2020. URL : http:// journals.openedition.org/cdg/507 ; DOI : https://doi.org/10.4000/cdg.507

\section{(c) (1) ()}

La revue Carnets de géographes est mise à disposition selon les termes de la Licence Creative Commons Attribution - Pas d'Utilisation Commerciale - Pas de Modification 4.0 International. 


\title{
BRADLEY L.GARRETT (2013), EXPLORE EVERYTHING : PLACE-HACKING THE CITY, Londres : Verso
}

\author{
LOUISE DORIGNON \\ ENS de Lyon \\ Trinity College (Université de Cambridge) \\ Géographie \\ louise.dorignon@ens-lyon.fr
}

Comment les lieux deviennent-ils des espaces de l'entre-deux ? En analysant I'utilisation temporaire d'objets urbains, la pratique provisoire de voies de circulation, ou le recours éphémère, transitoire, voire fugitif à des lieux défendus de la ville, l'ouvrage Explore Everything: Place-Hacking the City renseigne la catégorie des espaces de l'entre-deux par une analyse pertinente de la pratique contemporaine de l'exploration urbaine.

"Explore Everything ", le titre de l'ouvrage de Bradley Garrett, pourrait presque être la devise de ce groupe d'individus désignés comme explorateurs urbains (bien que la plupart refusent cette dénomination, comme toute autre affiliation) qui se retrouvent dans des métropoles mondiales afin de franchir les frontières interdites de la ville. Aussi les premières pages du récit constituent-elles une invitation à une vaste promenade pédestre, nocturne et à bout de souffle dans des espaces urbains aussi variés que Londres, Paris, Berlin, Detroit, Chicago, Las Vegas et Los Angeles. Le point commun entre ces villes que parcourent l'auteur et une communauté d'individus agiles et peu avares de leur sommeil est de renfermer des lieux et des espaces de circulation qui seraient secrets.

Ces espaces sont, pour la plupart des citadins, délaissés car inconnus ou invisibles, ignorés car d'accès restreint et limités par les frontières de la loi et/ou de la propriété privée, ou encore abandonnés après que la crise n'a rendu impossibles les activités qui s'y déroulaient auparavant. Pour les explorateurs urbains, dont l'auteur fait partie, la pratique de ces lieux ou de ces objets (il peut s'agir d'une grue) ouvre aussi une quête identitaire : l'expérience de ces franchissements urbains, qui sont autant de dépassements de soi, provoquerait leur épanouissement intérieur. L'auteur affirme en effet : "Urban explorers, much like computer 
hackers in virtual space, exploit fractures in the architecture of the city. Their goal is to find deeper meaning in the spaces we pass through every day. ${ }^{1}{ }^{1}(2013: 6)$.

"Explore Everything ", outre une invitation à " tout explorer ", devient alors un manifeste politique pour une liberté de circulation absolue dans la ville. L'ouvrage relate avec une plume précise et haletante différentes expéditions urbaines et questionne par-là même la notion de limites dans la ville: en repoussant les frontières imposées par la légalité, la sécurité ou l'usage que l'on donne aux objets et aux lieux, l'auteur mène une réflexion sur la finitude des espaces urbains et leur possible inachèvement. Ces espaces constituent en effet autant de terrains d'aventures et de jeu, mais aussi de creusets dans lesquels les individus expriment leur droit à la ville.

Ces lieux, aussi bien centraux que périphériques, ne constituent des espaces de l'entre-deux qu'en tant qu'ils sont franchis par ce groupe d'explorateurs, puisque c'est la pratique même de leur exploration qui les fait exister entre d'autres catégories d'espaces ouverts à la circulation libre et autorisée des individus. Sans ces expéditions, ces lieux resteraient des blancs, des vides; davantage des non-lieux que des espaces de transition. Pour Bradley Garrett, ce sont les pratiques sociales et spatiales de l'exploration (en anglais UE pour Urban Exploration) ou du place-hacking qui donnent corps à ces espaces, et qui les font exister ou survivre comme partie intégrante de nos villes.

En ouvrant son ouvrage par une citation de St Exupéry issue de Terres des Hommes (1949) "Mais quelle étrange leçon de géographie je reçus là! "- et en se faisant le narrateur, Bradley Garrett endosse avec lucidité et franchise le costume de l'ethnographe acteur de ses recherches. Il plonge alors le lecteur dans le réseau serré de son récit, bien tissé par quatre années d'explorations urbaines qui le conduisent à la publication d'un ouvrage profondément humain. À la fin de son bref prologue, l'auteur affirme :

"This is a story of friendships I forged over four years with a group that, despite severe consequences and repercussions, refused to let adventure, mystery and desire wither in a world rendered increasingly mundane by media saturation, gentrification, surveillance, the constrictions of civil liberties and health and safety laws" ${ }^{2}$ (2013 : xiv)

Le premier chapitre ( The UE Scene $»^{3}$ ) présente alors in medias res la communauté de l'UE à travers le récit de l'ascension du Shard, alors encore en construction dans la City de Londres, avant d'exprimer les grandes lignes de la philosophie de l'UE, ses inspirations (entre autres Guy Debord et le refus des impératifs du capitalisme contemporain), ses codes et ses modes de fonctionnements. C'est véritablement à travers une architecture souterraine et réticulaire que nous conduit l'auteur. On comprend vite que le but de ces explorations ne se

\footnotetext{
${ }^{1}$ Les explorateurs urbains, tels les pirates informatiques des espaces virtuels, exploitent les fractures dans l'architecture de la ville. Leur but est de trouver des significations plus profondes dans les espaces que nous traversons tous les jours.

${ }^{2}$ Ceci est I'histoire d'amitiés que j'ai forgées pendant quatre années avec un groupe qui, malgré de graves conséquences et répercussions, a refusé de laisser s'étioler l'aventure, le mystère et le désir dans un monde rendu de plus en plus banal par la saturation des médias, la gentrification, la surveillance, la restriction des libertés civiles et les lois sur la santé et la sécurité.

${ }^{3}$ La scène UE
} 
situe pas tant dans le résultat final que dans l'exploit lui-même : "The goal is more about the exploit itself. ${ }^{4}(2013: 19)$.

Ces explorations, si elles peuvent être interprétées comme des coups d'actions quasi politiques, tendent aussi à la fascination pour les espaces vides, abandonnés, et flirtent avec une esthétisation des ruines qui n'est pas sans rappeler le ruin porn dénoncé par certains géographes comme occultant les causalités qui ont mené à l'abandon de ces espaces (Paddeu, 2013). Le deuxième chapitre de l'ouvrage ("The Ruins of History " ${ }^{5}$ ) peut en cela constituer une justification de ces explorations. La mémoire involontaire serait le principal mécanisme qui attirerait les explorateurs dans des lieux abandonnés: "by visiting places that have been recently abandoned, we encounter artefacts of abandonment that are familiar to us and invoke spectres of unexpected and involuntary memories " ${ }^{6}(2013: 41)$. Par ailleurs, le livre foisonne de photographies en couleur qui montrent des perspectives de la ville à couper le souffle, et qui se veulent vertigineuses et poétiques. Il s'agit principalement de vues aériennes de la ville, présentant un skyline inédit dans un effort de se représenter soi-même les contours bien connus de la métropole londonienne, vegasienne ou los angélienne. Ces velléités frondeuses de créer sa propre perspective sur les gratte-ciels rejoignent les problématiques du skyline telles qu'elles ont été définies par Manuel Appert, " un enjeu de pouvoir et de construction identitaire en tant que modalité physique de territorialisations multiples passées, présentes et projetées " (2011: 7). Sur plus de la moitié de ces photos, les explorateurs urbains y sont mis en scène (de façon statique ou acrobatique), choix qui reflète peut-être la volonté de l'auteur de se prémunir contre les détracteurs du ruin porn.

La dimension humaine et sociale n'est jamais bien loin dans l'ouvrage de Bradley Garrett : au sein de son troisième chapitre ("Capturing Transition ${ }^{7}$ ), l'auteur se livre à une réflexion sur les effets psychologiques de la destruction de bâtiments chez ceux qui y ont vécus, travaillés, grandis etc. Loin de l'idée selon laquelle les explorateurs sont les auteurs de vandalismes, Bradley Garrett suggère que ces derniers contribuent aux efforts des historiens en protégeant et en documentant des sites fragiles qui recèlent pourtant des objets intéressants. Les chapitres suivants ("The rise of an infiltration crew »" et "Grails of the Underground ${ }^{9}$ ) sont davantage thématiques, et proposent d'une part la description des interactions au quotidien entre les explorateurs, craners $^{10}$ et autres night climbers et d'autre part le récit complet des aventures souterraines de ces explorateurs, dont la plupart se situent dans les réseaux de transports. À l'inverse, le sixième chapitre obéit à une logique régionale et se concentre sur les explorations aux États-Unis ( Hacking the New World ${ }^{11}$ ) : c'est l'occasion pour Bradley Garrett de revenir sur la dispute concernant Detroit, et le désormais fameux Michigan Central Station qui s'y situe.

\footnotetext{
${ }^{4}$ Le but réside plus dans l'exploit lui-même.

${ }^{5}$ Les ruines de l'histoire

${ }^{6}$ En visitant des lieux qui ont été récemment abandonnés, nous trouvons des objets qui nous ont été familiers et nous invoquons les spectres de l'inattendu et de la mémoire involontaire.

${ }^{7}$ Saisir la transition

${ }^{8}$ La montée d'une équipe d'infiltration

${ }^{9}$ Les graals des souterrains

${ }^{10}$ Ceux qui escaladent les grues

${ }^{11}$ Pirater le nouveau monde
} 
Ces explorations et les photos qui y sont prises, bien que secrètes, bénéficient d'une visibilité sur de nombreux blogs qui attire immanquablement les médias. Dans son dernier chapitre ( Crowds and cuffs » ${ }^{12}$ ), l'auteur revient sur le lien entre cette pratique et ses recherches en tant qu'ethnographe, ainsi que sur les raisons qui l'ont poussé à lever le voile sur l'exploration urbaine. Ses conversations avec les médias, son arrestation par la police britannique et son séjour en garde à vue pendant lequel l'épilogue du livre aurait été écrit sont relatés dans le détail.

Le livre de Bradley Garrett peut donc être lu comme une célébration et une protestation, " It is both a celebration and a protest » $(2013: 6)$, une volonté de réaffirmer sa liberté en empruntant des chemins jusque-là interdits, un désir de braver les limites de la ville autant que ses propres limites pour parvenir à des points de vue jamais considérés. La dimension contestataire est visiblement présente dans l'ouvrage, qui ne prétend jamais se départir de l'engagement qui préfigure à ces explorations : il s'agit bien de revendiquer la récupération de tous les espaces urbains, de faire tomber la censure sur les lieux ou l'homme ne pourrait véritablement accéder. Bradley Garrett invoque pour satisfaire à la question des risques et de la responsabilité, le libre-arbitre :

"I am not suggesting that one way of experiencing a place is superior to another. Indeed, many people would prefer to take a safe tour or sit in a climate-controlled viewing platform with a cocktail. But just as many people, I would venture to guess, would opt for adventure, given the choice, or would be happy to let others make that decision for themselves. Sadly, it is increasingly not a choice we are given. (...) The explorer reclaims rights to the city that are not on offer" ${ }^{13}$ (2013 : 236).

Dans le même temps, cette appropriation pionnière s'accompagne d'un véritable plaisir, plaisir qui motive inlassablement ce groupe hétérogène et insaisissable que sont les explorateurs urbains: celui de d'accéder à une autre essence de la ville, et par cette reconquête spatiale, de faire sienne la ville dans sa totalité.

Comme les romans à choix multiples, l'ouvrage semble offrir deux lectures, chacune correspondant à une lecture plus ou moins politisée. En cela, Bradley Garrett propose, à l'image des explorations urbaines, une lecture à ciel ouvert et une lecture plus souterraine de son ouvrage. À première vue, Explore Everything peut en effet être lu comme un roman d'aventure, dévoilant au fil des pages les plaisirs et les dangers associés à l'exploration de villes dont les représentations frôlent celles de cités interdites. Toutefois Explore Everything, est marqué par la pensée radicale de son éditeur, Verso, et une fois considéré comme un manifeste politique et libertaire, invite à une prise de position. Le lecteur se doit alors d'interpréter le manichéisme sous-jacent du livre (la vendetta pour la liberté d'un groupe

\footnotetext{
${ }^{12}$ Foules et menottes

${ }^{13}$ Je ne prétends pas qu'une façon de faire l'expérience d'un lieu est supérieure à une autre. En effet, beaucoup de gens préféreraient suivre une visite guidée ou s'asseoir sur une plateforme d'observation chauffée avec un cocktail. Mais comme beaucoup de gens, comme je me risque à l'imaginer, opteraient pour une aventure, s'ils avaient le choix, ou seraient heureux de laisser les autres décider pour eux-mêmes. (...) Malheureusement, on nous donne de moins en moins le luxe de choisir. (...) Les explorateurs s'approprient un droit à la ville qui ne leur est pas offert.
} 
d'explorateurs urbains, élevés au statut de quasi-justiciers, contre l'État répressif et arbitraire) non comme une simplification naïve de la réalité, mais bien comme un parti-pris politiquement engagé de l'auteur.

APPERT M. (2011), " Politique du skyline. Shard et le débat sur les tours à Londres ", Métropolitiques.

http://www.metropolitiques.eu/Politique-du-skyline-Shard-et-le.html (Consulté le 25/04/1014)

PADDEU F. (2013), " Portfolio, Detroit, In the D », Urbanités.

http://www.revue-urbanites.fr/portfolio-in-the-d/ (Consulté le 25/04/2014) 